\title{
Análisis del comportamiento mecánico de concreto tradicional y concreto con materiales reciclados.
}

\author{
E. Sánchez Murillo ${ }^{2}$ F. J. Olguin Coca ${ }^{1,2}$, L. D. López León ${ }^{1}$, I Castañeda Robles ${ }^{1}$, L. Lizárraga Mendiola ${ }^{1}$, F. \\ Almeraya Calderon ${ }^{3}$, J.A. Cabral Miramontes ${ }^{3}$, M. A. Baltazar Zamora ${ }^{4}$, J. J. Mejía Briseño², E. Hernández Perez ${ }^{2}$ \\ *olguinc@uaeh.edu.mx \\ ${ }^{1}$ CA Construcción Sostenible y Desarrollo de Materiales, AAI, Universidad Autónoma del Estado de Hidalgo, \\ Pachuca, México \\ ${ }^{2}$ Ciencias de la Tierra, Instituto Tecnologico de Pachuca, \\ ${ }^{3}$ CIIIA, Universidad Autónoma de Nuevo León, Monterrey, México \\ ${ }^{4}$ Facultad de Ingeniería, Universidad Veracruzana, Xalapa, México
}

\section{RESUMEN}

El concreto es un material 100\% reciclable, siempre que no esté contaminado, y puede utilizarse en muchas obras y estructuras diferentes cumpliendo con las especificaciones para los materiales necesarias. El concreto reciclado tiene su principal fuente de recursos en los residuos de construcción y demolición que proceden en su mayor parte de la demolición de edificios, pavimentos, excedentes en plantas concreteras, y como resultado urbanizaciones. Esto representa una pérdida de las materias primas que componen el concreto, principalmente del Agregado Natural (AN), ya que este es un recurso natural no renovable. Además, este desperdicio también implica la necesidad de un sitio para su disposición final. El concepto de construcción sostenible engloba la reducción, reutilización, y reciclaje de los residuos que genera la gran actividad de construcción. En este trabajo, se evalúa el desempeño de concretos fabricados con agregado reciclado(AR), a partir de cilindros de concreto con una resistencia especificada de $300 \mathrm{~kg} / \mathrm{cm} 2$. Las mezclas fueron elaboradas con 100\% de reemplazo del Agregado Grueso Natural (AGN), por Agregado Grueso Reciclado (AGR). Los resultados muestran una variación considerable en la prueba de absorción de agua ya que las mezclas fabricadas con el AR requieren mayor inclusión de agua, esto evidencia que las mezclas elaboradas con AGR propician un concreto mas poroso y por ende más susceptible desde el punto de vista de la durabilidad; aun cuando los niveles de resistencia son similares a los del concreto tradicional.

Palabras clave: concreto, comportamiento mecánico, reciclados.

\begin{abstract}
Concrete is a $100 \%$ recyclable material, as long as it is not contaminated, and can be used in many different works and structures meeting the specifications for the necessary materials. Recycled concrete has its main source of resources in construction and demolition waste that comes mostly from the demolition of buildings, pavements, surpluses in concrete plants, and as a result urbanizations. This represents a loss of raw materials that make up concrete, mainly Natural Aggregate (AN), since this is a non-renewable natural resource. In addition, this waste also implies the need for a site for its final disposal. The concept of sustainable construction encompasses the reduction, reuse, and recycling of waste generated by large construction activity. In this work, the performance of concretes manufactured with recycled aggregate (AR) is evaluated, from concrete cylinders with a specified resistance of $300 \mathrm{~kg} / \mathrm{cm} 2$. The mixtures were made with $100 \%$ replacement of Natural Coarse Aggregate (AGN), by Recycled Coarse Aggregate (AGR). The results show a considerable variation in the water absorption test since the mixtures manufactured with AR require greater inclusion of water, this shows that the mixtures made with AGR favor a more porous concrete and therefore more susceptible from the point of view of durability; even though the resistance levels are similar to those of traditional concrete.
\end{abstract}


Keywords: concrete, mechanical behavior, recycled.

\section{INTRODUCCIÓN}

El concreto hidráulico es un compuesto que resulta de la mezcla de material cementante, arena, grava, agua y en algunos casos aditivos, que al fraguar y endurecer adquieren propiedades similares a las de las rocas naturales más resistentes. Cuando se utiliza concreto hidráulico en las construcciones, éste tiene que ser diseñado y elaborado bajo un estricto control de calidad, conforme a parámetros de las normas aplicables en el país, como lo es las normas NMX, ASTM y en su caso los Comités ACI.

Es por esto que en este apartado, se abordan los principales temas sobre el concreto, como son: cada uno de los componentes de la mezcla y sus características, el comportamiento en estado fresco del concreto, las etapas de fraguado, las propiedades del concreto en estado endurecido y finalmente se hace una descripción breve de los métodos más comunes para el diseño de mezclas de concreto[1].

El concreto es un material 100\% reciclable, siempre que no esté contaminado, y puede utilizarse en muchas obras y estructuras diferentes cumpliendo con las especificaciones para los materiales necesarias. El concreto reciclado tiene su principal fuente de recursos en los residuos de construcción y demolición que proceden en su mayor parte de la demolición de edificios, pavimentos, excedentes en plantas concreteras, y como resultado de urbanizaciones. Esto representa una pérdida de las materias primas que componen el concreto, principalmente del Agregado Natural (AN), ya que este es un recurso natural no renovable. Además, este desperdicio también implica la necesidad de un sitio para su disposición final. El concepto de construcción sostenible engloba la reducción, reutilización, y reciclaje de los residuos que genera la gran actividad de construcción El empleo del concreto reciclado data de tiempos posteriores a la segunda guerra mundial, donde europeos enfrentaban la problemática de la gran acumulación de escombros en sus ciudades destruidas, motivo por el cual se abocaron a reciclar dichos desperdicios y utilizarlos como material de construcción, dándoles muy buenos resultados. Una investigación experimental en Estados Unidos, emprendida por [5] consistió en probar la resistencia a la fatiga de un material utilizado para base de pavimento, compuesto por agregados reciclados, pequeñas cantidades de cemento Pórtland y cenizas volantes, reforzado con fibra de acero. Las muestras preparadas alcanzaron límites de resistencia comparables con los materiales típicos utilizados en pavimentos para autopistas. En este mismo campo de la construcción, [7], de la Universidad Griffith en Australia, presentaron resultados de su trabajo con agregados reciclados de concreto (RCA) obtenidos por trituración. Los especímenes fueron sometidos a la prueba triaxial con repetición de carga un día después de su compactación.

Se determinó que la resistencia puede verse afectada significativamente debido al contenido de finos $(10 \%)$ en las muestras por lo que su utilización en bases y sub bases de carreteras queda sujeta a un control efectivo de estándares de calidad del producto.

Por su parte, Chini et al (2001) reportan desde la Universidad de Florida, una valuación de materiales reciclados de concreto para base de pavimento con mezclas asfálticas en caliente y como agregado de cemento Portland en pavimentos rígidos. Comprobaron que en la medida que aumenta el porcentaje de RCA en las muestras disminuye la resistencia a esfuerzos de flexión y compresión. Sin embargo los valores alcanzados, del orden de $35 \mathrm{Mpa}$, se consideran aceptables. Similares resultados se hallaron en Corea, presentados por Park [3] al experimentar con material reciclado seco y húmedo para bases de pavimento rígido, y en [6] quienes estudiaron las características de 
esfuerzo deformación de los agregados reciclados de concreto sometidos a compresión triaxial.

La fabricación de elementos de concreto con material reciclado de residuos de la construcción, puede tener el inconveniente de valores altos de porosidad redundando en problemas de durabilidad, principalmente por la penetración de cargas ambientales como son los cloruros y carbonatos.

El ACI 318-02 define la durabilidad del concreto como la "capacidad para resistir a la acción del tiempo, los ataques químicos, la abrasión o cualquier otro proceso de deterioro; es decir, que el concreto durable debe retener su forma original, su calidad y sus condiciones de servicio, cuando se exponga a su medio ambiente". Las condiciones de exposición que acarrean los daños más comunes en el concreto provienen de los líquidos y gases que lo penetran, originando acciones químicas que derivan en la corrosión del acero de refuerzo.

\section{PROCEDIMIENTO}

1. Identificación, de distintos laboratorios de construcción en la ciudad de Pachuca, Hidalgo, y sus áreas circundantes; La selección será de acuerdo a la ubicación y aceptación de los propietarios donde se encuentren los residuos.

2. Acopio de material (especímenes de concreto) en forma selectiva.

3. Obtención por cuarteo y secado de muestras de materiales pétreos heterogéneos y trituración de estos en una planta industrial de producción de agregados de la localidad.

4. Caracterización física del material triturado de acuerdo a la normatividad oficial para muestreo y pruebas de materiales, como son:

a) Peso volumétrico seco suelto para obtener el peso de las partículas sólidas por unidad de volumen, expresado en Kg. por metro cúbico.

b) Análisis granulométrico para clasificar por tamaño las partículas del material pasándolo por una sucesión de mallas, obteniendo el peso de los retenidos como porcentaje de la muestra total.

c) Densidad relativa, a obtener como la relación de la densidad absoluta, incluyendo sus vacíos.

d) Porcentaje de absorción, para determinar la cantidad de agua absorbida por el material pétreo previamente saturado en agua a una temperatura entre $15^{\circ}$ a $25^{\circ} \mathrm{C}$ durante 24 horas. e) Equivalente de arena, cuyo objeto será determinar en lo que pasa la malla número 4.75, bajo condiciones de pruebas establecidas, la proporción volumétrica de partículas de tamaño mayor que el de las arcillas, con respecto al volumen de las partículas finas de tamaño similar al de las citadas arcillas.

f) Porcentaje de desgaste, a fin de conocer la calidad del material pétreo en cuanto al grado de alteración y presencia de planos de debilitamiento que pudieran provocar una desintegración de las partículas.

Los resultados mostraron poca diferencia entre ambos, tal y como se observa en la tabla 1 siguiente:

Tabla 1 Características de los materiales

Característica

Unidades

Material

Natural

Reciclado 


\begin{tabular}{|ll|l|l|l}
\hline $\begin{array}{l}\text { Peso Vol. } \\
\text { Suelto }\end{array}$ & Seco & $\mathbf{K g} / \mathbf{m}^{3}$ & $\mathbf{1 0 6 0}$ & $\mathbf{1 1 2 6}$ \\
\hline $\begin{array}{l}\text { Peso Vol. } \\
\text { Comp }\end{array}$ & Seco & $\mathbf{K g} / \mathbf{m}^{3}$ & $\mathbf{1 1 5 2}$ & $\mathbf{1 1 8 1 . 5}$ \\
\hline $\begin{array}{l}\text { Densidad } \\
\text { Absorción }\end{array}$ & $\mathbf{K g} / \mathbf{L t}$ & $\mathbf{2 . 0 1}$ & $\mathbf{1 . 9 8}$ \\
\hline
\end{tabular}

5. Diseño de mezclas con el pétreo obtenido haciendo variar la relación agua- cemento para obtener especímenes con distintos valores de resistencia a la compresión, lo mas cercanos posibles a 300 $\mathrm{kg} / \mathrm{cm} 2$. con una nomenclatura como se muestra en la tabla 2 .

Tabla 2 Siglas de nomenclatura de especímenes

NOMENCLATURA

\begin{tabular}{|l|l|}
\hline $\mathbf{R}$ & Reciclado \\
\hline $\mathbf{N}$ & Natural \\
\hline AG & $\begin{array}{l}\text { Agregado } \\
\text { Grueso }\end{array}$ \\
\hline AF & Agregado Fino \\
\hline $\mathbf{2 0}$ & Porcentaje \\
\hline
\end{tabular}

6. Pruebas de Resistencia a la compresión. Se efectuará en forma tradicional de acuerdo a la norma ASTM C 39-03 a 14 y 28.

7. Integración del informe final y conclusiones

En la figura 1 se muestra el cribado del material en base a la norma ASTM para la elaboración de los especímenes de concreto empleando los residuos de concreto después del triturado del material. 

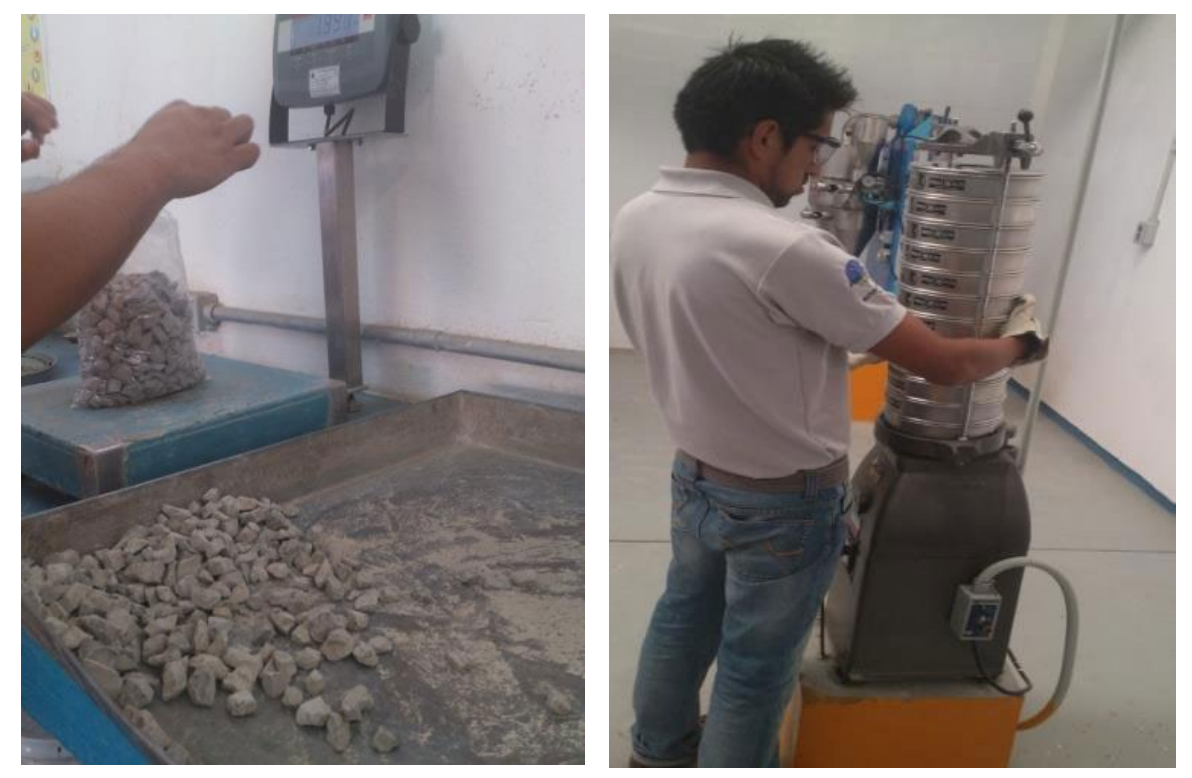

Figura 1 Cribado de materiales

\section{RESULTADOS}

En la Grafica 1 se muestran los resultados de compresion de todas las mezclas realizadas de los especimenes experimentados a los 3, 14 y 28 dias de curado donde se muestra que el comportamiento de materiales con agregado grueso reciclado presentan resistencias a la compresión con valores cercanos a la resistencia de diseño, qlos que se realizaron con agregado fino reciclado presentaron un comportamiento mecanico con un $71 \%$ de la resistencia de diseño y donde los datos con agregado natural presenta la mejor resistencia a la compresión debido a que el agregado grueso tiene un mejor desempeño como agregado mineral grueso presentando una la resistencia de diseño al igual una mejor mezcla con los materiales.

\section{Resistencia a la Compresión}

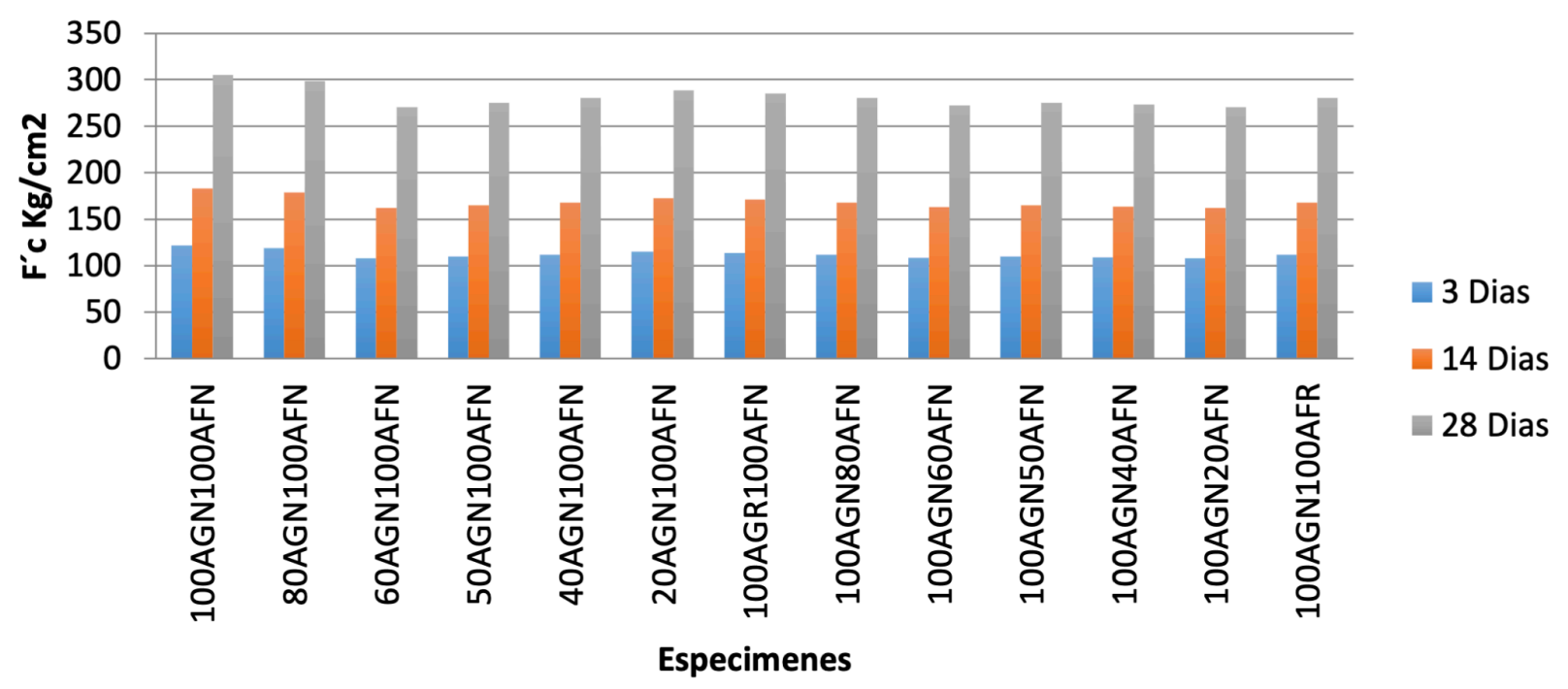

Grafica 1 Resistencia a la compresión 
En la grafica 2 se muestra el comportamiento de los especimenes con agregado grueso reciclado donde se observa que el $20 \%$ de AGR presenta una resistencia del 85\% de la resistencia de diseño y conforme aumenta el AGR en porcentaje la resistencia decrece por lo que de manera preliminar la resistencia tiene un optimo con el 20\%de AGR, las resistencias presentadas con los porcentajes superiores al $20 \%$ sus resistencias esntan en un promedio del $75 \%$ de la resistencia de diseño.

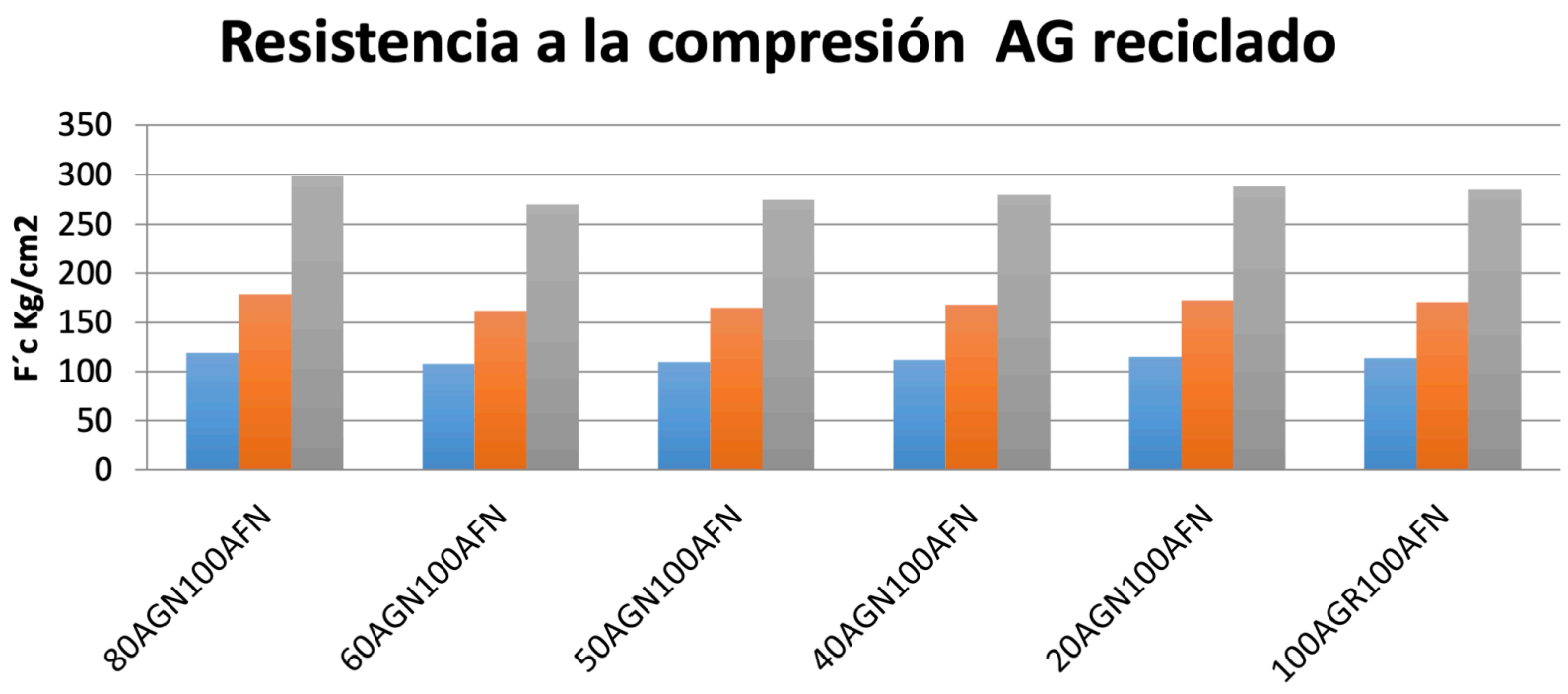

Especimenes

Grafica 2 Resistencia a la compresión de agregado grueso reciclado

Los resultados del AFR en sus diferentes porcentajes muestran un promedio de $78 \%$ del $\mathrm{f}^{\prime} \mathrm{c}$ de diseño los resultados en mínima proporción que mejoran su comportamiento mecánico son con $20 \%$ y $100 \%$ de AFR el cambio no es tan significativo debido a que el AFR no aporta la resistencia mecánica del concreto, mejora su comportamiento pero no es el que aporta ese comportamiento mecánico.

\section{Resistencia a la compresión AF reciclado}

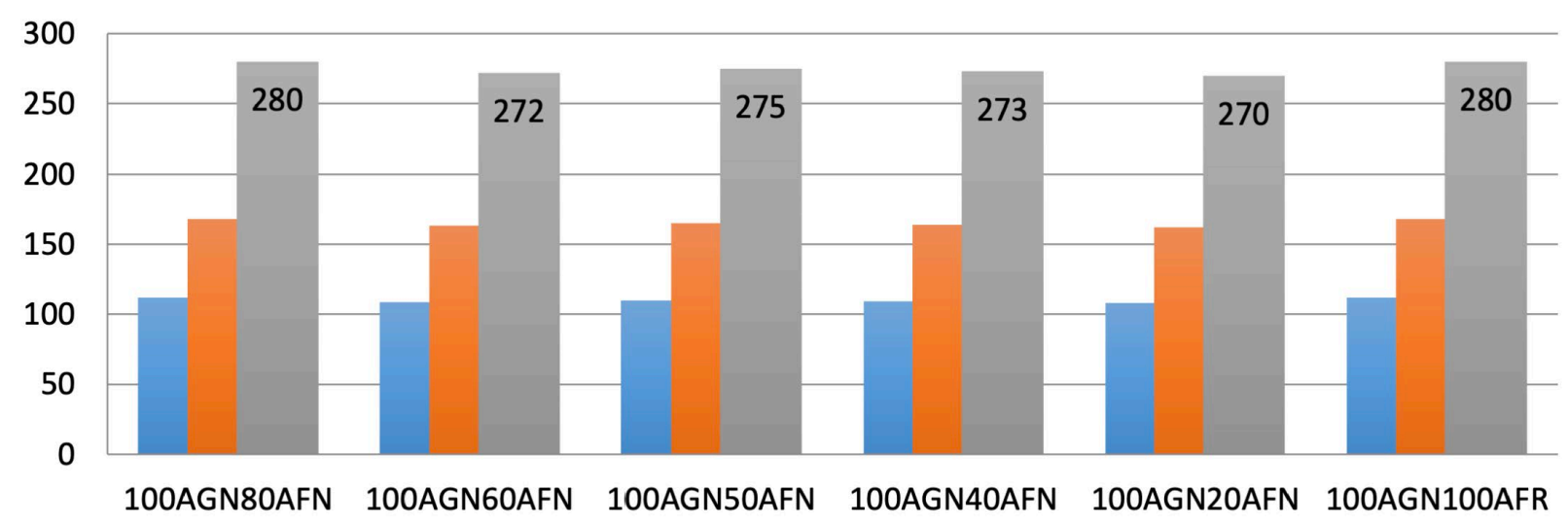

Grafica 3 Resistencia a la compresión de agregado fino reciclado 


\section{CONCLUSIONES}

La resietencia a la compresion de materiales reciclados no muestran una gran predida de la resistencia en comparación con los agregados naturales, tambien se observa un mejor comportamiento con agregados reciclados gruesos en comparación con los finos.

Los agregados reciclados gruesos presentan un comportamiento mecánico semejante a los agregados naturales el agregado reciclado grueso como tal es muy resistente y debido a su forma genera una buena cohesión con los materiales al realizar la mezcla originando una mejor resistencia a la compresión.

Los agregados finos no presentaron resultados hipoteticos donde se pensaba que el fino reaccionaria con el cemento y generaria una mayor dosificacion de cemento lo cual no se presento así, sindo que no aumenta la resistencia y presentan resultados de compresion menores que un agregado fino natural.

Los materiales reciclados de concreto pueden emplearse en elementos estrcturales siendo que los resultados muestran que no existen gran variación con agregados naturales.

\section{AGRADECIMIENTOS}

Éstos deberán citar a los individuos, organizaciones o instituciones que prestaron algún apoyo especial en el desarrollo del trabajo

\section{REFERENCIAS}

[1] Michel , A., Solgaard , A., \& Pease , B. J. (2013). Experimental investigation of the relation between damage at the concrete-steel interface and initiation of reinforcement corrosion in plain and fibre reinforced concrete. Corrosion Science , 77, 308-321.

[2] Krykowskia , T., \& Zybura, A. (2013). Modelling of Reinforced Concrete Element Damage as a Result of Reinforcement Corrosion. Procedia Engineering , 57, 614 - 623. México, I. d. (2006).

[3] Retama V.J. y Ayala M.G., C. 2. (2013). Reinforced concrete modelling using enriched finite elements. CFRAC 2013 The third international conference on computational modelling of fracture and failure of materials and structures.

[4] Yuxi Zhao, J. D. (2014). Steel corrosion and corrosion-induced cracking in recycled aggregate concrete. Corrosion Science, 85, 241-250.

[5] DURAR, O. T. (1998. ). Manual de inspección, Evaluación y Diagnostico de Corrosión en Estructuras de Hormigón Armado. Maracaibo, Venezuela: CYTED.

[6] Anuario estadístico de la minería en el Estado de'México, 2005. Mexico, Mexico, Mexico: Gobierno del Estado de México. 
[7] Baltazar-Zamora, M. A. (2005). Comportamiento de la corrosión en acero al carbono e inoxidable embebido en concreto en presencia de $\mathrm{NaCl}$ y Na2SO4. Chihuahua, Chih, México: CIMAV. 